\title{
A graph associated to a lattice
}

\author{
Mojgan Afkhami · Zahra Barati • \\ Kazem Khashyarmanesh
}

Received: 29 May 2013 / Revised: 1 July 2013 / Published online: 10 August 2013

(C) The Author(s) 2013. This article is published with open access at Springerlink.com

\begin{abstract}
In this paper, we associate a simple graph to a lattice $\mathcal{L}$, in which the vertex set is being the set of all elements of $\mathcal{L}$, and two distinct vertices $x$ and $y$ are adjacent if $x \vee y \in S$, when $S$ is a multiplicatively closed subset of $\mathcal{L}$. We denote this graph by $\Gamma_{S}(\mathcal{L})$. We study some properties of $\Gamma_{S}(\mathcal{L})$. Moreover, we investigate the planarity of $\Gamma_{S}(\mathcal{L})$, whenever $S$ is a saturated multiplicatively closed subset of $\mathcal{L}$.
\end{abstract}

Keywords Lattice $\cdot$ Planar graph

Mathematics Subject Classification (2000) $\quad$ 05C10 06B99

\section{Introduction}

The study of algebraic structures, using the properties of graph theory, tends to an exciting research topic in the last decade. There are many papers on assigning a graph to a ring, see for example $[2,3,6,4]$. One of these graphs is the total graph. Let $R$ be a commutative ring. The total graph of $R$ is a simple graph with vertex set $R$, and two distinct vertices $a$ and $b$ are adjacent if $a+b$ is a zero divisor of $R$ (see for

Communicated by Salvatore Rionero.

M. Afkhami

Department of Mathematics, University of Neyshabur, 91136-899 Neyshabur, Iran

e-mail: mojgan.afkhami@yahoo.com

Z. Barati · K. Khashyarmanesh $(\varangle)$

Department of Pure Mathematics, Ferdowsi University of Mashhad, 1159-91775 Mashhad, Iran

e-mail: khashyar@ipm.ir

Z. Barati

e-mail: za.barati87@gmail.com 
example [1,2]). By using this idea, for a multiplicatively closed subset $S$ of $R$, in [5], the authors defined a graph on $R$, denoted by $\Gamma_{S}(R)$, with vertices as elements of $R$, and two distinct vertices $a$ and $b$ are adjacent if and only if $a+b \in S$. In this paper, we generalize the concept of $\Gamma_{S}(R)$ for a lattice. Recall that a lattice is an algebra $\mathcal{L}=(L, \vee, \wedge)$ satisfying the following conditions: for all $a, b, c \in L$,

1. $a \wedge a=a, a \vee a=a$,

2. $a \wedge b=b \wedge a, a \vee b=b \vee a$,

3. $(a \wedge b) \wedge c=a \wedge(b \wedge c),(a \vee b) \vee c=a \vee(b \vee c)$, and

4. $a \vee(a \wedge b)=a \wedge(a \vee b)=a$.

There is an equivalent definition for a lattice (see for example [8, Theorem 2.1]). To do this, for a lattice $\mathcal{L}$, one can define an order $\leqslant$ on $\mathcal{L}$ as follows: For any $a, b \in \mathcal{L}$, we set $a \leq b$ if and only if $a \wedge b=a$. Then $(\mathcal{L}, \leq)$ is an ordered set in which every pairs of elements has a greatest lower bound (g.l.b.) and a least upper bound (l.u.b.). Conversely, let $P$ be an ordered set such that, for every pair $a, b \in P$, g.l.b. $(a, b) \in P$ and l.u.b. $(a, b) \in P$. For each $a$ and $b$ in $P$, we define $a \wedge b:=$ g.l.b. $(a, b)$ and $a \vee b:=$ l.u.b. $(a, b)$. Clearly $(P, \wedge, \vee)$ is a lattice.

The lattice $\mathcal{L}$ is said to be bounded if there are elements 0 and 1 such that

$$
0 \wedge x=0 \text { and } x \vee 1=1
$$

for all $x \in \mathcal{L}$. Let $S$ be a nonempty subset of $\mathcal{L}$. We say that $S$ is a multiplicatively closed subset of $\mathcal{L}$ if $x \wedge y \in S$, for all $x$ and $y$ of $S$. Also, we say that a subset $S$ of $\mathcal{L}$ is saturated if $x \wedge y \in S$ if and only if $x, y \in S$.

For a multiplicatively closed subset of $\mathcal{L}$, we define $\Gamma_{S}(\mathcal{L})$ as a simple graph, with vertex-set $\mathcal{L}$ and two distinct vertices $x$ and $y$ being adjacent if and only if $x \vee y \in S$.

In the second section, we study some basic properties of the graph $\Gamma_{S}(\mathcal{L})$ such as connectivity, diameter and completeness whenever $\mathcal{L}$ is a bounded lattice. In the third section, for bounded lattices, we investigate $\Gamma_{S}(\mathcal{L})$, whenever $S$ is a saturated multiplicatively closed subset of $\mathcal{L}$ and, in the final section, we study the planarity of $\Gamma_{S}(\mathcal{L})$ where $\mathcal{L}$ is a bounded lattice.

Now, we start to remind a belief necessary background of lattice theory from [8]. Let $x$ and $y$ be two distinct elements of $\mathcal{L}$. Whenever $x \leqslant y$ and there is no element $z$ in $\mathcal{L}$ such that $x \leqslant z \leqslant y$, we say y covers $x$. An element $a \in \mathcal{L}$ is an atom of lattice $\mathcal{L}$ if it covers 0 . Also, an element $m \in \mathcal{L}$ is a coatom of lattice $\mathcal{L}$ if 1 covers it. We denote the set of all coatoms of $\mathcal{L}$ by Coatom $(\mathcal{L})$ and the set of atoms of $\mathcal{L}$ by Atom $(\mathcal{L})$. An ideal $I$ of $\mathcal{L}$ is a non-empty subset of $\mathcal{L}$ such that

(i) for all $a$ and $b$ of $I, a \vee b \in I$, and

(ii) for any $a \in I$ and $b \in \mathcal{L}, a \wedge b \in I$.

An ideal $I$ of $\mathcal{L}$ is maximal if $I$ is proper $(I \neq \mathcal{L})$ and the only ideal having $I$, as a proper subset, is $\mathcal{L}$. We use the notation $\mathrm{J}(\mathcal{L})$ for the jacobson radical of $\mathcal{L}$ which is the intersection of all maximal ideals of $\mathcal{L}$. Given a lattice $\mathcal{L}$ and $A \subseteq \mathcal{L}$. An element $x \in \mathcal{L}$ is a lower bound of $A$ if $x \leq a$ for all $a \in A$. An upper bound is defined in a dual mannar. The set of all lower bounds of $A$ is denoted by $A^{\ell}$ and the set of all upper bounds by $A^{u}$, where 


$$
A^{\ell}:=\{x \in \mathcal{L} ; x \leq a \text { for all } a \in A\}
$$

and

$$
A^{u}:=\{x \in \mathcal{L} ; a \leq x \text { for all } a \in A\} .
$$

Now, we recall some definitions of graph theory from [7] which are needed in this paper. The degree of a vertex $v$ in the graph $X$ is the number of edges of $X$ incident with $v$ and denoted by $\operatorname{deg}(v)$. In a graph $X$ with vertex-set $V(X)$, the distance between two distinct vertices $a$ and $b$, denoted by $\mathrm{d}(a, b)$, is the length of a shortest path connecting $a$ and $b$, if such a path exists; otherwise, we set $\mathrm{d}(a, b):=\infty$. The diameter of a graph $X$ is $\operatorname{diam}(X)=\sup \{\mathrm{d}(a, b): a$ and $b$ are distinct vertices of $X$ \}. For two distinct vertices $a$ and $b$ in $X$, the notation $a-b$ means that $a$ and $b$ are adjacent. Also the girth of a graph $X$, denoted by $\operatorname{gr}(X)$, is the length of a shortest cycle in $X$ if $X$ has a cycle; otherwise, we set $\operatorname{gr}(X):=\infty$. A graph $X$ is said to be connected if there exists a path between any two distinct vertices, and it is complete if it is connected with diameter one. We use $K_{n}$ to denote the complete graph with $n$ vertices. Also, we say that $X$ is totally disconnected if no two vertices of $X$ are adjacent. A clique of a graph is a complete subgraph of $X$ and the number of vertices in a largest clique of $G$ is called the clique number of $G$ and is denoted by $\omega(G)$.

\section{Basic properties of $\Gamma_{S}(\mathcal{L})$}

Form now on $\mathcal{L}$ is a bounded lattice. In this section $S$ is a multiplicatively closed subset of $\mathcal{L}$. We begin with the following lemma.

Lemma 2.1 If $0 \notin S$, then $|S \cap \operatorname{Atom}(\mathcal{L})| \leq 1$.

Proof Assume in contrary that $|S \cap \operatorname{Atom}(\mathcal{L})| \geq 2$. Let $a_{1}$ and $a_{2}$ be two distinct elements in $S \cap \operatorname{Atom}(\mathcal{L})$. Since $S$ is a multiplicatively closed subset of $\mathcal{L}$, we have $a_{1} \wedge a_{2} \in S$ which implies that $0 \in S$. This is a required contradiction. Hence $|S \cap \operatorname{Atom}(\mathcal{L})| \leq 1$.

Lemma 2.2 The following statements hold.

(i) If $0 \in S$, then $\operatorname{deg}(0)=|S|-1$. Otherwise, $\operatorname{deg}(0)=|S|$.

(ii) If $1 \in S$, then $\operatorname{deg}(1)=|\mathcal{L}|-1$. Otherwise, $\operatorname{deg}(1)=0$.

Proof Since $0 \vee x=x$ and $1 \vee x=1$, for all $x \in \mathcal{L}$, the statements (i) and (ii) hold.

In the following theorem, we study the connectivity of $\Gamma_{S}(\mathcal{L})$.

Theorem 2.3 $\Gamma_{S}(\mathcal{L})$ is connected if and only if $1 \in S$. Moreover, if $\Gamma_{S}(\mathcal{L})$ is connected, then $\operatorname{diam}\left(\Gamma_{S}(\mathcal{L})\right) \leq 2$. 
Proof Suppose that $1 \in S$. By part (ii) of Lemma 2.2, $\operatorname{deg}(1)=|\mathcal{L}|-1$ which implies that $\Gamma_{S}(\mathcal{L})$ is connected.

Conversely, if $\Gamma_{S}(\mathcal{L})$ is connected, then $\operatorname{deg}(1) \neq 0$. Now, by part (ii) of Lemma 2.2, we have $1 \in S$.

The last statement is clear.

Proposition 2.4 $\Gamma_{S}(\mathcal{L})$ is complete if and only if $S=\mathcal{L}$ or $S=\mathcal{L} \backslash\{0\}$.

Proof Assume that $\Gamma_{S}(\mathcal{L})$ is complete. So $\operatorname{deg}(0)=|\mathcal{L}|-1$. By part (i) of Lemma 2.2, we have $|S|=|\mathcal{L}|-1$ or $|S|-1=|\mathcal{L}|-1$. Hence $S=\mathcal{L}$ or $S=\mathcal{L} \backslash\{x\}$, for some $x \in \mathcal{L}$. We claim that $x=0$. Assume in contrary that $x \neq 0$. Since $0 \vee x=x$, the vertex 0 is not adjacent to $x$, which is impossible. So $x=0$, and thus $S=\mathcal{L}$ or $S=\mathcal{L} \backslash\{0\}$.

The converse statement follows easily.

Theorem 2.5 The following statements hold.

(i) The graph $\Gamma_{S}(\mathcal{L})$ is regular if and only if it is either complete or totally disconnected.

(ii) $\Gamma_{S}(\mathcal{L})$ is a star graph if and only if $S=\{1\}$ or $S=\{1,0\}$ and $|\operatorname{Coatom}(\mathcal{L})|=1$.

Proof (i) Suppose that $\Gamma_{S}(\mathcal{L})$ is regular and that is not totally disconnected. By part (ii) of Lemma 2.2, we have $1 \in S$. Then $\operatorname{deg}(1)=|\mathcal{L}|-1$. Since $\Gamma_{S}(\mathcal{L})$ is regular, $\operatorname{deg}(x)=|\mathcal{L}|-1$, for all $x \in \mathcal{L}$. Hence $\Gamma_{S}(\mathcal{L})$ is complete.

The converse statement is clear.

(ii) Suppose that $\Gamma_{S}(\mathcal{L})$ is a star graph. Since $\Gamma_{S}(\mathcal{L})$ is connected, we have $1 \in S$, and so $\operatorname{deg}(1)=|\mathcal{L}|-1$. Hence 1 is the center of $\Gamma_{S}(\mathcal{L})$. On the other hand, since 0 is adjacent to all element of $S$, we have $S=\{1\}$ or $S=\{1,0\}$. Assume in contrary that $|\operatorname{Coatom}(\mathcal{L})| \geq 2$. Thus there exists distinct vertices $m$ and $m^{\prime}$ in Coatom $(\mathcal{L})$ which are adjacent in $\Gamma_{S}(\mathcal{L})$. This is impossible. Hence $|\operatorname{Coatom}(\mathcal{L})|=1$.

The converse statement is clear.

Proposition 2.6 Assume that $1 \in S$. Then $\operatorname{gr}\left(\Gamma_{S}(\mathcal{L})\right) \in\{3, \infty\}$.

Proof Suppose that $|\operatorname{Coatom}(\mathcal{L})| \geq 2$ and consider the cycle $1-m-m^{\prime}-1$ in $\Gamma_{S}(\mathcal{L})$, where $m, m^{\prime} \in \operatorname{Coatom}(\mathcal{L})$, to deduce that $\operatorname{gr}\left(\Gamma_{S}(\mathcal{L})\right)=3$. Otherwise, $|\operatorname{Coatom}(\mathcal{L})|=$ 1. Now we have the following cases:

(i) Suppose that $|S|=1$. Then $S=\{1\}$ and so, by Proposition 2.5, the graph $\Gamma_{S}(\mathcal{L})$ is a star graph. Thus $\operatorname{gr}\left(\Gamma_{S}(\mathcal{L})\right)=\infty$.

(ii) Suppose that $|S|=2$. If $S=\{0,1\}$, then, by Proposition $2.5, \Gamma_{S}(\mathcal{L})$ is a star graph and so $\operatorname{gr}\left(\Gamma_{S}(\mathcal{L})\right)=\infty$. Otherwise, $S=\{1, s\}$ where $s \neq 0$. Now, the cycle $1-s-0-1$ is the shortest cycle in the graph $\Gamma_{S}(\mathcal{L})$ which implies that $\operatorname{gr}\left(\Gamma_{S}(\mathcal{L})\right)=3$.

(iii) Suppose that $|S| \geq 2$. Thus there is $s \in S$ such that $s \neq 0,1$. Now, the cycle $1-s-0-1$ is the shortest cycle in the graph $\Gamma_{S}(\mathcal{L})$. So $\operatorname{gr}\left(\Gamma_{S}(\mathcal{L})\right)=3$.

Let $\mathcal{A}$ be a chain in $S$. Since $x \vee y \in S$ for all $x, y \in \mathcal{A}$, we have the following proposition.

Proposition $2.7 \omega\left(\Gamma_{S}(\mathcal{L})\right) \geq \max \{|\mathcal{A}| ; \mathcal{A}$ is a chain in $S\}$. 


\section{Basic properties of $\Gamma_{S}(\mathcal{L})$ where $S$ is a saturated subset of the lattice $\mathcal{L}$}

Throughout this section, $S$ is a saturated subset of the lattice $\mathcal{L}$. It is easy to see that if $x \in S$, then $\{x\}^{u} \subseteq S$, and so we always have $1 \in S$. Hence, by Proposition 2.3, one can conclude that $\Gamma_{S}(\mathcal{L})$ is connect with diameter less than three.

Proposition 3.1 For all $s \in S$, we have that $\operatorname{deg}(\mathrm{s})=|\mathcal{L}|-1$.

Proof Let $s$ be an arbitrary element in $S$. Since $s \wedge(s \vee x)=s$ for all $x \in \mathcal{L}$, we have that $s \wedge(s \vee x) \in S$. Therefore $\operatorname{deg}(\mathrm{s})=|\mathcal{L}|-1$ for all $s \in S$.

In the following proposition, we present a lower bound for the clique number of $\Gamma_{S}(\mathcal{L})$.

Proposition 3.2 In the graph $\Gamma_{S}(\mathcal{L})$ we have the following inequality.

$$
\omega\left(\Gamma_{S}(\mathcal{L})\right) \geq \max \{|S|,|\operatorname{Coatom}(\mathcal{L})|\}+1
$$

Proof Since $m \vee m^{\prime}=1$ for all $m$ and $m^{\prime}$ in $\operatorname{Coatom}(\mathcal{L})$, the set $\operatorname{Coatom}(\mathcal{L}) \cup\{1\}$ forms a clique in $\Gamma_{S}(\mathcal{L})$. Also, by Proposition 3.1, $S \cup\{0\}$ is a clique in $\Gamma_{S}(\mathcal{L})$. This implies that $\omega\left(\Gamma_{S}(\mathcal{L})\right) \geq \max \{|S|,|\operatorname{Coatom}(\mathcal{L})|\}+1$.

Lemma 3.3 Assume that $0 \in S$. Then $\Gamma_{S}(\mathcal{L})$ is complete.

Proof Since $0 \in S$, we have that $\{0\}^{u} \subseteq S$. Hence $S=\mathcal{L}$. Now, by Proposition 2.4, the graph $\Gamma_{S}(\mathcal{L})$ is complete.

From now on, we assume that $0 \notin S$. So, by Lemma 2.1, we have $|S \cap \operatorname{Atom}(\mathcal{L})| \leq 1$.

Proposition 3.4 If $S \cap \operatorname{Atom}(\mathcal{L})=\{a\}$, then $S=\{a\}^{u}$.

Proof Clearly $\{a\}^{u} \subseteq S$. Now, assume in contrary that there exists $b \in S \backslash\{a\}^{u}$. Since $S$ is a multiplicatively closed subset of $\mathcal{L}$, we have $0=a \wedge b \in S$, which is impossible. Thus $S=\{a\}^{u}$.

Proposition 3.5 Suppose that $|\mathcal{L}| \geq 3$.

(i) If $|S| \geq 2$, then every vertex of the graph $\Gamma_{S}(\mathcal{L})$ lies in a cycle of length 3 , and so $\operatorname{gr}\left(\Gamma_{S}(\mathcal{L})\right)=3$.

(ii) If $|S|=1$, then $\operatorname{gr}\left(\Gamma_{S}(\mathcal{L})\right) \in\{3, \infty\}$.

Proof (i) Since $|S| \geq 2$, we can choose $s \neq 1$ in $S$. Now, let $x$ be an arbitrary element in $\mathcal{L}$. Then we have the cycle $1-x-s-1$, and so each vertex of the graph $\Gamma_{S}(\mathcal{L})$ lies in a cycle of length 3 and $\operatorname{gr}\left(\Gamma_{S}(\mathcal{L})\right)=3$.

(ii) Since $|S|=1$, we have that $S=\{1\}$. The result now follows from Proposition 2.6 .

Proposition 3.6 If $|S|=1$, then $\operatorname{deg}(x)=1$, for all $x \in J(\mathcal{L})$. 
Proof Since $|S|=1$, we have that $S=\{1\}$. If $|\operatorname{Coatom}(\mathcal{L})|=1$, then, by Proposition $2.5, \Gamma_{S}(\mathcal{L})$ is a star graph with center 1 . Since $1 \notin J(\mathcal{L})$, every vertex in $\mathrm{J}(\mathcal{L})$ has degree one. Otherwise, $|\operatorname{Coatom}(\mathcal{L})| \geq 2$. Let $x$ be an arbitrary vertex in $J(\mathcal{L})$. Clearly 1 is adjacent to every vertex in $\Gamma_{S}(\mathcal{L})$ which implies that $\operatorname{deg}(x) \geq 1$. Assume in contrary that $\operatorname{deg}(x) \geq 2$. So there is $y \neq 1$ in $\mathcal{L}$ such that $x$ and $y$ are adjacent, and so $x \vee y=1$. If $y \in \operatorname{Coatom}(\mathcal{L})$, then $x \vee y=y$, which is impossible. Hence $y \notin \operatorname{Coatom}(\mathcal{L})$, but there is $m \in \operatorname{Coatom}(\mathcal{L})$ such that $y \in\{m\}^{\ell}$. Since $x \in \cap_{m \in \operatorname{Coatom}(\mathcal{L})}\{m\}^{\ell}$, we have that $x \vee y \leq m$, which is impossible. So $\operatorname{deg}(x)=1$, for all $x \in J(\mathcal{L})$.

Proposition 3.7 If $|S|=1$ and $|\operatorname{Coatom}(\mathcal{L})| \geq 2$, then every vertex in graph $\Gamma_{S}(\mathcal{L} \backslash$ $\mathrm{J}(\mathcal{L})$ ) lies in a cycle of length 3 .

Proof Since $|S|=1$, we have that $S=\{1\}$. Let $y$ be an arbitrary element in $\mathcal{L} \backslash J(\mathcal{L})$. We need to show that $y$ lies in a cycle of length 3 in $\Gamma_{S}(\mathcal{L} \backslash J(\mathcal{L}))$. If $y \in \operatorname{Coatom}(\mathcal{L})$, since $|\operatorname{Coatom}(\mathcal{L})| \geq 2$, there exists $m$ with $m \neq y$ in Coatom $(\mathcal{L})$. Thus, we can consider the cycle $y-m-1-y$ in $\Gamma_{S}(\mathcal{L} \backslash J(\mathcal{L}))$. Otherwise $y \notin \operatorname{Coatom}(\mathcal{L})$. Since $y \notin J(\mathcal{L})$, there exists $m \in \operatorname{Coatom}(\mathcal{L})$ such that $y \notin\{m\}^{\ell}$. Therefore $y \vee m=1$, which implies that $y$ and $m$ are adjacent in $\Gamma_{S}(\mathcal{L} \backslash J(\mathcal{L}))$. So we can consider the cycle $y-m-1-y$ and the result follows.

\section{Planarity of $\Gamma_{S}(\mathcal{L})$ when $S$ is a saturated subset of $\mathcal{L}$}

In this section, we characterize all planar graphs $\Gamma_{S}(\mathcal{L})$, where $S$ is a saturated subset of $\mathcal{L}$. Recall that a planar graph is a graph that can be embedded on the plane, that is, it can be drawn on the plane in such a way that it's edges intersect only at their endpoints. Kuratowski provided a nice characterization of planar graphs, which now is known as Kuratowski's Theorem:

A finite graph is planar if and only if it does not contain a subdivision of $K_{5}$ or $K_{3,3}$.

Proposition 4.1 If $0 \in S$, then $\Gamma_{S}(\mathcal{L})$ is planar if and only if $|\mathcal{L}| \leq 4$.

Proof If $0 \in S$, then $S=\mathcal{L}$. Hence $\Gamma_{S}(\mathcal{L})$ is a complete graph, and so the result follows from Kuratowski's Theorem.

Proposition 3.2 in conjunction with Kuratowski's Theorem implies the following lemma.

Lemma 4.2 If $|\operatorname{Coatom}(\mathcal{L})| \geq 4$ or $|S| \geq 4$, then $\Gamma_{S}(\mathcal{L})$ is not planar.

In view of Lemma 4.2, we investigate to study the planarity of $\Gamma_{S}(\mathcal{L})$, in the case that $|S| \leqslant 3$ and $|\operatorname{Coatom}(\mathcal{L})| \leqslant 3$. We start with the following proposition.

Proposition 4.3 Suppose that $|S|=3$. Then $\Gamma_{S}(\mathcal{L})$ is planar if and only if $|\mathcal{L}| \leq 5$.

Proof Suppose that $\Gamma_{S}(\mathcal{L})$ is planar. Assume in contrary that $|\mathcal{L}| \geq 6$. Now, put $V_{1}:=S$ and $V_{2}:=\left\{x_{1}, x_{2}, x_{3}\right\} \subseteq \mathcal{L} \backslash S$. Clearly one can find a copy of $K_{3,3}$ in $\Gamma_{S}(\mathcal{L})$. 
Therefore, by Kuratowski's Theorem, $\Gamma_{S}(\mathcal{L})$ is not planar, which is impossible. Hence $|\mathcal{L}| \leq 5$.

Conversely, assume that $|\mathcal{L}| \leq 5$. It is clear that if $|\mathcal{L}| \leq 4$, then $\Gamma_{S}(\mathcal{L})$ is planar. Also, if $|\mathcal{L}|=5$, then, in view of Proposition $2.4, \Gamma_{S}(\mathcal{L})$ is not $K_{5}$. So $\Gamma_{S}(\mathcal{L})$ is planar.

Note that, if $|S|=2$, then $S=\{1, m\}$ for some $m \in \operatorname{Coatom}(\mathcal{L})$. Also, if $|S|=1$, then $S=\{1\}$.

For the rest of the paper, we need the following definition.

Definition 4.4 Let $x$ be an arbitrary element in $\mathcal{L}$. We define the lower neighbors of $x$ as the set $B_{\ell}(x):=\{y \in \mathcal{L} ; x$ covers $y\}$. Also, for every subsets $A$ and $B$ of $\mathcal{L}$, we put $L_{A}^{B}:=\{B\}^{\ell} \backslash\{A\}^{\ell}$. Moreover, for $x \in \mathcal{L}$, we denote the sets $L_{A}^{\{x\}}$ and $L_{\{x\}}^{B}$, by $L_{A}^{x}$ and $L_{x}^{B}$, respectively.

Lemma 4.5 Assume that $S=\{1, m\}$, where $m \in \operatorname{Coatom}(\mathcal{L})$. If $\left|B_{\ell}(m)\right| \geq 3$, then $\Gamma_{S}(\mathcal{L})$ is not planar.

Proof Since $\left|B_{\ell}(m)\right| \geq 3$, we can choose the subset $A=\left\{x_{1}, x_{2}, x_{3}\right\}$ of the set $B_{\ell}(m)$. It is clear that $x_{i} \vee x_{j}=m$ for all $1 \leq i \neq j \leq 3$. So, the induced subgraph of $\Gamma_{S}(\mathcal{L})$ on $A \cup S$ is isomorphic to $K_{5}$. Thus, by Kuratowski's Theorem, $\Gamma_{S}(\mathcal{L})$ is not planar.

Proposition 4.6 Let $S=\{1, m\}$, where $m \in \operatorname{Coatom}(\mathcal{L})$. If $\Gamma_{S}(\mathcal{L})$ is planar, then one of the following statements holds:

(i) $\left|B_{\ell}(m)\right|=1$,

(ii) $\left|B_{\ell}(m)\right|=2$, and $\left|L_{x}^{y}\right| \leq 2$, for all $x, y \in B_{\ell}(m)$, and if $\left|L_{x}^{y}\right|=2$, then $\left|L_{y}^{x}\right|=1$, and if $\left|L_{y}^{x}\right|=2$, then $\left|L_{x}^{y}\right|=1$.

Proof Since $\Gamma_{S}(\mathcal{L})$ is planar, by Lemma 4.5, $\left|B_{\ell}(m)\right| \leq 2$. Suppose that $\left|B_{\ell}(m)\right| \neq 1$. Thus, we have that $\left|B_{\ell}(m)\right|=2$. Set $B_{\ell}(m):=\{x, y\}$. If $\left|L_{y}^{x}\right| \geq 3$ or $\left|L_{x}^{y}\right| \geq 3$, then it is easy to see that $\Gamma_{S}(\mathcal{L})$ has a subgraph which is isomorphic to $K_{3,3}$, and so $\Gamma_{S}(\mathcal{L})$ is not planar, which is impossible. Hence $\left|L_{y}^{x}\right| \leq 2$ and $\left|L_{x}^{y}\right| \leq 2$.

Now, suppose that $\left|L_{x}^{y}\right|=2$ and $\left|L_{y}^{x}\right|=2$. Set $V_{1}:=L_{x}^{y} \cup\{m\}$ and $V_{2}:=L_{y}^{x} \cup\{1\}$. It is easy to see that $\Gamma_{S}(\mathcal{L})$ has a subgraph isomorphic to $K_{3,3}$ with parts $V_{1}$ and $V_{2}$, which is impossible. So if $\left|L_{x}^{y}\right|=2$, then $\left|L_{y}^{x}\right|=1$. Also, if $\left|L_{y}^{x}\right|=2$, then $\left|L_{x}^{y}\right|=1$.

Assume that $S=\{1, m\}$, where $m \in \operatorname{Coatom}(\mathcal{L})$. By Lemma 4.2, we have $\mid$ Coatom $(\mathcal{L}) \mid \leq 3$. In the following proposition, we present a necessary and sufficient condition for planarity of $\Gamma_{S}(\mathcal{L})$ whenever $|\operatorname{Coatom}(\mathcal{L})|=1$.

Theorem 4.7 Let $S=\{1, m\}$ and $\operatorname{Coatom}(\mathcal{L})=\{m\}$. Then the graph $\Gamma_{S}(\mathcal{L})$ is planar if and only if one of the conditions (i) or (ii) in Proposition 4.6 holds.

Proof Let $\Gamma_{S}(\mathcal{L})$ is planar. Then, by Proposition 4.6, we are done.

Conversly, we show that if one of the conditions (i) or (ii) in Proposition 4.6 occurs, then $\Gamma_{S}(\mathcal{L})$ is planar. One can easily check that, if $\left|B_{\ell}(m)\right|=1$, then $\Gamma_{S}(\mathcal{L})$ is isomorphic to $K_{1,1,|\mathcal{L}|-2}$, and so $\Gamma_{S}(\mathcal{L})$ is planar. Also, if condition (ii) in Proposition 4.6 occurs, then it is not hard to see that $\Gamma_{S}(\mathcal{L})$ is planar. 
In the next theorem, we provide a necessary and sufficient condition for planarity of $\Gamma_{S}(\mathcal{L})$ whenever $\mid$ Coatom $(\mathcal{L}) \mid=2$.

Theorem 4.8 Suppose that $S=\{1, m\}$ and $\operatorname{Coatom}(\mathcal{L})=\left\{m, m^{\prime}\right\}$. Then the graph $\Gamma_{S}(\mathcal{L})$ is planar if and only if $\mathcal{L}$ is one of the following lattices:

(i) $\mathcal{L}=S \cup L_{m^{\prime}}^{x} \cup \mathrm{J}(\mathcal{L})$, where $B_{\ell}(m)=\{x\}$ such that $x \in\left\{m^{\prime}\right\}^{\ell}$.

(ii) $\mathcal{L}=S \cup L_{x}^{m^{\prime}} \cup L_{m^{\prime}}^{x} \cup \mathrm{J}(\mathcal{L})$, where $B_{\ell}(m)=\{x\}$ such that

(a) $x \notin\left\{m^{\prime}\right\}^{\ell}$.

(b) The numbers $\left|L_{x}^{m^{\prime}}\right|$ and $\left|L_{m^{\prime}}^{x}\right|$ are less than 3, and if $\left|L_{x}^{m^{\prime}}\right|=2$, then $\left|L_{m^{\prime}}^{x}\right|=1$, and if $\left|L_{m^{\prime}}^{x}\right|=2$, then $\left|L_{x}^{m^{\prime}}\right|=1$.

(iii) $\mathcal{L}=S \cup\left\{m^{\prime}\right\} \cup B_{\ell}(m) \cup \mathrm{J}(\mathcal{L})$, where $\left|B_{\ell}(m)\right|=2$ and $\left|B_{\ell}(m) \backslash\left\{m^{\prime}\right\}^{\ell}\right|=1$.

Proof Assume that $\Gamma_{S}(\mathcal{L})$ is a planar graph. Then, by Lemma 4.6, $\left|B_{\ell}(m) \backslash\left\{m^{\prime}\right\}^{\ell}\right| \leq 2$. Now, suppose that $\left|B_{\ell}(m) \backslash\left\{m^{\prime}\right\}^{\ell}\right|=2$, and set $B_{\ell}(m) \backslash\left\{m^{\prime}\right\}^{\ell}:=\{x, y\}$. Since the induced subgraph of $\Gamma_{S}(\mathcal{L})$ on $\left\{1, m, m^{\prime}, x, y\right\}$ is isomorphic to $K_{5}$, by Kuratowski's Theorem, $\Gamma_{S}(\mathcal{L})$ is not planar, which is impossible. Thus $\left|B_{\ell}(m) \backslash\left\{m^{\prime}\right\}^{\ell}\right| \leq 1$. Also, by Proposition 4.6, one of the conditions (i) or (ii) in Proposition 4.6 must holds. So we have the following cases:

Case 1. Suppose that the condition (i) in Proposition 4.6 holds and put $B_{\ell}(m):=\{x\}$. At first note that $\mathcal{L}=S \cup L_{x}^{m^{\prime}} \cup L_{m^{\prime}}^{x} \cup \mathrm{J}(\mathcal{L})$. Now, assume that $\left|B_{\ell}(m) \backslash\left\{m^{\prime}\right\}^{\ell}\right|=1$. Thus $B_{\ell}(m) \backslash\left\{m^{\prime}\right\}^{\ell}=\{x\}$. If $\left|L_{x}^{m^{\prime}}\right| \geq 3$, then it is easy to see that the graph $\Gamma_{S}(\mathcal{L})$ has a copy of $K_{3,3}$, which is impossible. Therefore $\left|L_{m}^{m^{\prime}}\right| \leq 2$. Similarly $\left|L_{m^{\prime}}^{x}\right| \leq 2$. Now, if $\left|L_{x}^{m^{\prime}}\right|=\left|L_{m^{\prime}}^{x}\right|=2$, then consider the sets $V_{1}:=L_{x}^{m^{\prime}} \cup\{1\}$ and $V_{2}:=L_{m^{\prime}}^{x} \cup\{m\}$, to deduce that $\Gamma_{S}(\mathcal{L})$ has a copy of $K_{3,3}$, which is impossible. Therefore, if $\left|L_{x}^{m^{\prime}}\right|=2$, then $\left|L_{m^{\prime}}^{x}\right|=1$. Similarly, if $\left|L_{m^{\prime}}^{x}\right|=2$, then $\left|L_{x}^{m^{\prime}}\right|=1$. So $\mathcal{L}=S \cup L_{x}^{m^{\prime}} \cup L_{m^{\prime}}^{x} \cup \mathrm{J}(\mathcal{L})$, where $B_{\ell}(m)=\{x\}$ and $\left|L_{x}^{m^{\prime}}\right|$, and $\left|L_{m^{\prime}}^{x}\right|$ are less than 3 , and if $\left|L_{x}^{m^{\prime}}\right|=2$, then $\left|L_{m^{\prime}}^{x}\right|=1$, and also, if $\left|L_{x}^{m^{\prime}}\right|=2$, then $\left|L_{m^{\prime}}^{x}\right|=1$.

Now, let $\left|B_{\ell}(m) \backslash\left\{m^{\prime}\right\}^{\ell}\right|=0$. Since $\left|B_{\ell}(m) \backslash\left\{m^{\prime}\right\}^{\ell}\right|=0$, we have that $L_{m^{\prime}}^{x}=\emptyset$. So $\mathcal{L}=S \cup L_{x}^{m^{\prime}} \cup \mathrm{J}(\mathcal{L})$.

Case 2. Suppose that the condition (ii) in Proposition 4.6 holds. Set $B_{\ell}(m):=\{x, y\}$. Note that in this case, we have that

$$
\mathcal{L}=S \cup L_{\{x, y\}}^{m^{\prime}} \cup L_{\left\{m^{\prime}, y\right\}}^{x} \cup L_{\left\{m^{\prime}, x\right\}}^{y} \cup L_{y}^{\left\{m^{\prime}, x\right\}} \cup L_{x}^{\left\{m^{\prime}, y\right\}} \cup L_{m^{\prime}}^{\{x, y\}} \cup \mathrm{J}(\mathcal{L}) .
$$

Since $\mathcal{L}$ is a lattice, we have that $\left|B_{\ell}(m) \backslash\left\{m^{\prime}\right\}^{\ell}\right|=1$. Without loss of generality, we may assume that $B_{\ell}(m) \backslash\left\{m^{\prime}\right\}^{\ell}=\{y\}$. If $\left|L_{\{x, y\}}^{m^{\prime}}\right| \geq 2$, then, by setting $V_{1}:=L_{\{x, y\}}^{m^{\prime}} \cup\{y\}$ and $V_{2}:=\{1, m, x\}$, one can find a copy of $K_{3,3}$ in the graph $\Gamma_{S}(\mathcal{L})$, which is impossible. Hence $L_{\{x, y\}}^{m^{\prime}}=\left\{m^{\prime}\right\}$. Since $x \in\left\{m^{\prime}\right\}^{\ell}$, we have that $L_{\left\{y, m^{\prime}\right\}}^{x}=\emptyset$. Also, if $\left|L_{\left\{x, m^{\prime}\right\}}^{y}\right| \geq 2$, then one can easily to find a copy of $K_{3,3}$, which is impossible. So $L_{\left\{x, m^{\prime}\right\}}^{y}=\{y\}$. On the other hand, since $x \in\left\{m^{\prime}\right\}^{\ell}, L_{m^{\prime}}^{\{x, y\}}=\emptyset$. Thus $\mathcal{L}=S \cup\left\{m^{\prime}\right\} \cup B_{\ell}(m) \cup \mathrm{J}(\mathcal{L})$.

The converse statment is clear.

In the following, we investigate the planarity of the graph $\Gamma_{S}(\mathcal{L})$ whenever $|S|=2$ and $\operatorname{Coatom}(\mathcal{L})=\left\{m, m^{\prime}, m^{\prime \prime}\right\}$. 
Proposition 4.9 Suppose that $S=\{1, m\}$, Coatom $(\mathcal{L})=\left\{m, m^{\prime}, m^{\prime \prime}\right\}$ and that the graph $\Gamma_{S}(\mathcal{L})$ is planar. Then the following statements hold.

(i) $L_{\left\{m^{\prime}, m^{\prime \prime}\right\}}^{m}=\{m\}$,

(ii) $\left|L_{\left\{m, m^{\prime \prime}\right\}}^{m^{\prime}}\right| \leq 2$ and $\left|L_{\left\{m, m^{\prime}\right\}}^{m^{\prime \prime}}\right| \leq 2$, and if $\left|L_{\left\{m, m^{\prime \prime}\right\}}^{m^{\prime}}\right|=2$ then $\left|L_{\left\{m, m^{\prime}\right\}}^{m^{\prime \prime}}\right|=1$, and if $\left|L_{\left\{m, m^{\prime}\right\}}^{m^{\prime \prime}}\right|=2$, then $\left|L_{\left\{m, m^{\prime \prime}\right\}}^{m^{\prime}}\right|=1$

Proof (i) Suppose that $\Gamma_{S}(\mathcal{L})$ is planar. Assume in contrary that $\left|L_{\left\{m^{\prime}, m^{\prime \prime}\right\}}^{m}\right| \geq 2$. Note that, for every element $x \in L_{\left\{m^{\prime}, m^{\prime \prime}\right\}}^{m}$, we have $x \vee m^{\prime}=1$ and $x \vee m^{\prime \prime}=1$. So the graph $\Gamma_{S}(\mathcal{L})$ has a copy of $K_{5}$, which is impossible. Thus $L_{\left\{m^{\prime}, m^{\prime \prime}\right\}}^{m}=\{m\}$.

(ii) Assume in contrary that $\left|L_{\left\{m, m^{\prime \prime}\right\}}^{m^{\prime}}\right| \geq 3$ or $\left|L_{\left\{m, m^{\prime}\right\}}^{m^{\prime \prime}}\right| \geq 3$. One can easily see that the graph $\Gamma_{S}(\mathcal{L})$ has a subgraph isomorphic to $K_{3,3}$, and so $\Gamma_{S}(\mathcal{L})$ is not planar, which is impossible. Therefore $\left|L_{\left\{m, m^{\prime \prime}\right\}}^{m^{\prime}}\right| \leq 2$ and $\left|L_{\left\{m, m^{\prime}\right\}}^{m^{\prime \prime}}\right| \leq 2$. Now, if $\left|L_{\left\{m, m^{\prime \prime}\right\}}^{m^{\prime}}\right|=2$ and $\left|L_{\left\{m, m^{\prime}\right\}}^{m^{\prime \prime}}\right|=2$, then, by setting $V_{1}:=L_{\left\{m, m^{\prime}\right\}}^{m^{\prime \prime}} \cup\{1\}$ and $V_{2}:=L_{\left\{m, m^{\prime \prime}\right\}}^{m^{\prime}} \cup\{m\}$, we can find a copy of $K_{3,3}$ in the graph $\Gamma_{S}(\mathcal{L})$, which is impossible. Hence if $\left|L_{\left\{m, m^{\prime \prime}\right\}}^{m^{\prime}}\right|=2$, then $\left|L_{\left\{m, m^{\prime}\right\}}^{m^{\prime \prime}}\right|=1$. Similarly if $\left|L_{\left\{m, m^{\prime}\right\}}^{m^{\prime \prime}}\right|=2$, then $\left|L_{\left\{m, m^{\prime \prime}\right\}}^{m^{\prime}}\right|=1$.

Theorem 4.10 Let $S=\{1, m\}$ and $\operatorname{Coatom}(\mathcal{L})=\left\{m, m^{\prime}, m^{\prime \prime}\right\}$. Then the graph $\Gamma_{S}(\mathcal{L})$ is planar if and only if $\mathcal{L}$ is one of the following lattices:

(i) $\mathcal{L}=\left\{1, m, m^{\prime}, m^{\prime \prime}, x\right\} \cup L_{x}^{\left\{m^{\prime}, m^{\prime \prime}\right\}} \cup \mathrm{J}(\mathcal{L})$, where $B_{\ell}(m)=\{x\}$ and $x \in$ $\left\{m^{\prime}\right\}^{\ell} \backslash\left\{m^{\prime \prime}\right\}^{\ell}$ or $x \in\left\{m^{\prime \prime}\right\}^{\ell} \backslash\left\{m^{\prime}\right\}^{\ell}$.

(ii) $\mathcal{L}=\{1, m, x\} \cup L_{\left\{x, m^{\prime \prime}\right\}}^{m^{\prime}} \cup L_{\left\{x, m^{\prime \prime}\right\}}^{m^{\prime}} \cup L_{x}^{\left\{m^{\prime}, m^{\prime \prime}\right\}} \cup \mathrm{J}(\mathcal{L})$, where $B_{\ell}(m)=\{x\}$ and

(a) $x \in\left\{m^{\prime}\right\}^{\ell} \cap\left\{m^{\prime \prime}\right\}^{\ell}$,

(b) $\left|L_{\left\{x, m^{\prime \prime}\right\}}^{m^{\prime}}\right| \leq 2$ and $\left|L_{\left\{x, m^{\prime \prime}\right\}}^{m^{\prime}}\right| \leq 2$, and if $\left|L_{\left\{x, m^{\prime \prime}\right\}}^{m^{\prime}}\right|=2$, then $\left|L_{\left\{x, m^{\prime}\right\}}^{m^{\prime \prime}}\right|=1$, and if $\left|L_{\left\{x, m^{\prime}\right\}}^{m^{\prime \prime}}\right|=2$, then $\left|L_{\left\{x, m^{\prime \prime}\right\}}^{m^{\prime}}\right|=1$

Proof Suppose that $\Gamma_{S}(\mathcal{L})$ is planar. Firstly, by Proposition 4.9 , we have $L_{\left\{m^{\prime}, m^{\prime \prime}\right\}}^{m}=$ $\{m\}$ which implies that $\left|B_{\ell}(m) \backslash\left\{m^{\prime}, m^{\prime \prime}\right\}^{\ell}\right|=0$. Now, by Proposition 4.6, we have the following cases:

Case 1. Suppose that the condition (i) of Proposition 4.6 holds. Then set $B_{\ell}(m):=\{x\}$. Since $\left|B_{\ell}(m) \backslash\left\{m^{\prime}, m^{\prime \prime}\right\}^{\ell}\right|=0$, we have the following subcases:

(a) $B_{\ell}(m) \backslash\left\{m^{\prime}\right\}^{\ell}=\{x\}$ and $B_{\ell}(m) \backslash\left\{m^{\prime \prime}\right\}^{\ell}=\emptyset$. In this case, if $\left|L_{\left\{x, m^{\prime \prime}\right\}}^{m^{\prime}}\right| \geq 2$, then one can easily find a copy of $K_{3,3}$, which is impossible. So $\left|L_{\left\{x, m^{\prime \prime}\right\}}^{m^{\prime}}\right|=1$. Similarly $\left|L_{\left\{x, m^{\prime}\right\}}^{m^{\prime \prime}}\right|=1$. Also $L_{\left\{m^{\prime}, m^{\prime \prime}\right\}}^{x}, L_{m^{\prime \prime}}^{\left\{x, m^{\prime}\right\}}$ and $L_{m^{\prime}}^{\left\{x, m^{\prime \prime}\right\}}$ are empty sets, because $x \in\left\{m^{\prime}\right\}^{\ell}$. So

$$
\mathcal{L}=\left\{1, m, m^{\prime}, m^{\prime \prime}, x\right\} \cup L_{x}^{\left\{m^{\prime}, m^{\prime \prime}\right\}} \cup \mathrm{J}(\mathcal{L})
$$

(b) $B_{\ell}(m) \backslash\left\{m^{\prime \prime}\right\}^{\ell}=\{x\}$ and $B_{\ell}(m) \backslash\left\{m^{\prime}\right\}^{\ell}=\emptyset$. It is similar to (a).

(c) $B_{\ell}(m) \backslash\left\{m^{\prime \prime}\right\}^{\ell}=\varnothing$ and $B_{\ell}(m) \backslash\left\{m^{\prime}\right\}^{\ell}=\emptyset$. In this case, by Proposition 4.9, we have that $\left|L_{\left\{x, m^{\prime \prime}\right\}}^{m^{\prime}}\right| \leq 2$ and $\left|L_{\left\{x, m^{\prime \prime}\right\}}^{m^{\prime}}\right| \leq 2$, and if $\left|L_{\left\{x, m^{\prime \prime}\right\}}^{m^{\prime}}\right|=2$, then $\left|L_{\left\{x, m^{\prime}\right\}}^{m^{\prime \prime}}\right|=1$ 
and vis versa. Also, since $x \in\left\{m^{\prime}\right\}^{\ell}$ and $x \in\left\{m^{\prime \prime}\right\}^{\ell}$, the sets $L_{\left\{m^{\prime}, m^{\prime \prime}\right\}}^{x}, L_{m^{\prime \prime}}^{\left\{x, m^{\prime}\right\}}$ and $L_{m^{\prime}}^{\left\{x, m^{\prime}\right\}}$ are empty. Thus $\mathcal{L}=\{1, m, x\} \cup L_{\left\{x, m^{\prime \prime}\right\}}^{m^{\prime}} \cup L_{\left\{x, m^{\prime \prime}\right\}}^{m^{\prime}} \cup L_{x}^{\left\{m^{\prime}, m^{\prime \prime}\right\}} \cup \mathrm{J}(\mathcal{L})$, where $\left|L_{\left\{x, m^{\prime \prime}\right\}}^{m^{\prime}}\right| \leq 2$ and $\left|L_{\left\{x, m^{\prime \prime}\right\}}^{m^{\prime}}\right| \leq 2$, and if $\left|L_{\left\{x, m^{\prime \prime}\right\}}^{m^{\prime}}\right|=2$ and $\left|L_{\left\{x, m^{\prime}\right\}}^{m^{\prime \prime}}\right|=1$ and if $\left|L_{\left\{x, m^{\prime}\right\}}^{m^{\prime \prime}}\right|=2$ and $\left|L_{\left\{x, m^{\prime \prime}\right\}}^{m^{\prime}}\right|=1$

Case 2. Suppose that the condition (ii) of Proposition 4.6 holds. Set $B_{\ell}(m):=\{x, y\}$. Since $\mathcal{L}$ is a lattice and $\left|B_{\ell}(m) \backslash\left\{m^{\prime}, m^{\prime \prime}\right\}^{\ell}\right|=0$, one can conclude that $\mid B_{\ell}(m) \backslash$ $\left\{m^{\prime}\right\}^{\ell} \mid=1$ and $\left|B_{\ell}(m) \backslash\left\{m^{\prime \prime}\right\}^{\ell}\right|=1$. So, without loss the generality, we may assume that $x \in L_{m^{\prime \prime}}^{m^{\prime}}$ and $y \in L_{m^{\prime}}^{m^{\prime \prime}}$. Now if we set $V_{1}:=\left\{1, m^{\prime}, x\right\}$ and $V_{1}:=\left\{m, m^{\prime \prime}, y\right\}$, then one can find a subgraph isomorphic to $K_{3,3}$ in $\Gamma_{S}(\mathcal{L})$ which is impossile. So this case never happens.

The converse statment is clear.

Now, the only remaining case is that $|S|=1$. The following lemmas are useful.

Lemma 4.11 Assume that $|S|=1$. Then the graph $\Gamma_{S}(\mathcal{L})$ is planar if and only if $\Gamma_{S}(\mathcal{L} \backslash J(\mathcal{L}))$ is planar.

Proof By Proposition 3.6 we have that $\operatorname{deg}(x)=1$, for all $x \in J(\mathcal{L})$. So, the result holds.

Lemma 4.12 Let $|S|=1$ and $|\operatorname{Coatom}(\mathcal{L})| \geq 2$. If $\left|L_{\operatorname{Coatom}(\mathcal{L}) \backslash\{m\}}^{m}\right| \geq 3$ and $\left|L_{m}^{m^{\prime}}\right| \geq$ 2 , for some distinct $m, m^{\prime} \in \operatorname{Coatom}(\mathcal{L})$, then $\Gamma_{S}(\mathcal{L})$ is not planar.

Proof Since $\left|L_{\text {Coatom }(\mathcal{L}) \backslash\{m\}}^{m}\right| \geq 3$, we set $V_{1}:=\left\{m, x_{1}, x_{2}\right\} \subseteq L_{\text {Coatom }(\mathcal{L}) \backslash\{m\}}^{m}$. Also, since $\left|L_{m}^{m^{\prime}}\right| \geq 2$, we set $V_{2}:=\left\{1, m^{\prime}, y_{1}\right\}$ where $y_{1} \in L_{m}^{m^{\prime}}$. It is easy to see that the subgraph of $\Gamma_{S}(\mathcal{L})$ on $V_{1} \cup V_{2}$ is isomorphic to $K_{3,3}$. Thus, by Kuratowski's Theorem, $\Gamma_{S}(\mathcal{L})$ is not planar.

In the next theorem, we provide a necessary and sufficient condition for planarity of $\Gamma_{S}(\mathcal{L})$ when $|S|=1$.

Theorem 4.13 Suppose that $|S|=1$. Then the graph $\Gamma_{S}(\mathcal{L})$ is planar if and only if one of the following statements holds:

(i) $|\operatorname{Coatom}(\mathcal{L})|=1$,

(ii) $|\operatorname{Coatom}(\mathcal{L})|=2$ and if there is an element $m \in \operatorname{Coatom}(\mathcal{L})$ such that $\left|L_{\text {Coatom }(\mathcal{L}) \backslash\{m\}}^{m}\right| \geq 3$, then $\left|L_{m}^{\text {Coatom }(\mathcal{L}) \backslash\{m\}}\right|=1$

(iii) $|\operatorname{Coatom}(\mathcal{L})|=3$, and

(a) For all $m \in \operatorname{Coatom}(\mathcal{L}),\left|L_{\text {Coatom }(\mathcal{L}) \backslash\{m\}}^{m}\right| \leq 2$ and there is at most one element $m$ in Coatom $(\mathcal{L})$ such that $\left|L_{\text {Coatom }(\mathcal{L}) \backslash\{m\}}^{m}\right|=2$.

(b) If there is $m \in \operatorname{Coatom}(\mathcal{L})$ such that $\left|L_{\operatorname{Coatom}(\mathcal{L}) \backslash\{m\}}^{m}\right|=2$, then $\left|L_{m}^{\text {Coatom }(\mathcal{L}) \backslash\{m\}}\right|=0$.

Proof Assume that the graph $\Gamma_{S}(\mathcal{L})$ is planar. Then, by Proposition 3.2, $|\operatorname{Coatom}(\mathcal{L})| \leq 3$. Suppose that $|\operatorname{Coatom}(\mathcal{L})| \neq 1$. So, we have the following cases: 
Case 1. Suppose that $|\operatorname{Coatom}(\mathcal{L})|=2$ and put $\operatorname{Coatom}(\mathcal{L})=\left\{m, m^{\prime}\right\}$. If there is one element $c \in \operatorname{Coatom}(\mathcal{L})$ such that $\left|L_{\operatorname{Coatom}(\mathcal{L}) \backslash\{c\}}^{c}\right| \geq 3$, then we show that $\left|L_{c}^{\text {Coatom }(\mathcal{L}) \backslash\{c\}}\right|=1$. Without loss the generality, we may assume that $c=m$. Assume in contrary that $\left|L_{m}^{m^{\prime}}\right| \geq 2$. Then, by setting $V_{1}:=\left\{1, m^{\prime}, x\right\}$ and $V_{2}:=\left\{m, y_{1}, y_{2}\right\}$, where $x \in L_{m}^{m^{\prime}}$ and $y_{1}, y_{2} \in L_{\text {Coatom }}^{m}(\mathcal{L}) \backslash\{m\}$, one can find a copy of $K_{3,3}$ in $\Gamma_{S}(\mathcal{L})$, which is impossible. Therefore if there is one element $m \in \operatorname{Coatom}(\mathcal{L})$ such that $\left|L_{\text {Coatom }(\mathcal{L}) \backslash\{m\}}^{m}\right| \geq 3$, then $L_{m}^{m^{\prime}}=\left\{m^{\prime}\right\}$.

Case 2. Suppose that $|\operatorname{Coatom}(\mathcal{L})|=3$ and put $\operatorname{Coatom}(\mathcal{L})=\left\{m, m^{\prime}, m^{\prime \prime}\right\}$. It is easy to see that if $\left|L_{\text {Coatom }(\mathcal{L}) \backslash\{m\}}^{m}\right| \geq 3$, then the graph $\Gamma_{S}(\mathcal{L})$ is not planar, which is impossible. So $\left|L_{\operatorname{Coatom}(\mathcal{L}) \backslash\{m\}}^{m}\right| \leq 2$ for all $m \in \operatorname{Coatom}(\mathcal{L})$. Now, Assume in contrary that $\left|L_{\text {Coatom }(\mathcal{L}) \backslash\{c\}}^{c}\right|=2$ and $\left|L_{\text {Coatom }(\mathcal{L}) \backslash\left\{c^{\prime}\right\}}^{c^{\prime}}\right|=2$ for some $c$ and $c^{\prime}$ in Coatom $(\mathcal{L})$. Without loss of generality, we may assume that $c=m$ and $c^{\prime}=m^{\prime}$. Put $V_{1}:=\{1, m, x\}$ and $V_{2}:=\left\{m^{\prime}, m^{\prime \prime}, y\right\}$, where $x \in L_{\left\{m^{\prime}, m^{\prime \prime}\right\}}^{m}$ and $y \in L_{\left\{m, m^{\prime \prime}\right.}^{m^{\prime}}$. Then one can find a subgraph of $\Gamma_{S}(\mathcal{L})$ which is isomorphic to $K_{3,3}$, which is impossible. So, there is at most one element $c$ in $\operatorname{Coatom}(\mathcal{L})$ such that $\left|L_{\operatorname{Coatom}(\mathcal{L}) \backslash\{c\}}^{c}\right|=2$. Now, assume that $\left|L_{\text {Coatom }(\mathcal{L}) \backslash\{m\}}^{m}\right|=2$, and assume in contrary that $\left|L_{m}^{\left\{m^{\prime}, m^{\prime \prime}\right\}}\right| \geq 1$. Then, by setting $V_{1}:=\{1\} \cup L_{\text {Coatom }(\mathcal{L}) \backslash\{m\}}^{m}$ and $V_{2}:=\left\{m^{\prime}, m^{\prime \prime}, y\right\}$ where $y \in L_{m}^{\left\{m^{\prime}, m^{\prime \prime}\right\}}$, we can find a subgraph in the graph $\Gamma_{S}(\mathcal{L})$ which is isomorphic to $K_{3,3}$, which is impossible. So, $\left|L_{m}^{\left\{m^{\prime}, m^{\prime \prime}\right\}}\right|=0$.

Conversely, if $|\operatorname{Coatom}(\mathcal{L})|=1$, then, by part (ii) of Proposition $2.5, \Gamma_{S}(\mathcal{L})$ is a star graph, and so it is planar.

Now, assume that $|\operatorname{Coatom}(\mathcal{L})|=2$ and that there is one element $m \in \operatorname{Coatom}(\mathcal{L})$ such that $\left|L_{\text {Coatom }(\mathcal{L}) \backslash\{m\}}^{m}\right| \geq 3$. Then $\left|L_{m}^{\operatorname{Coatom}(\mathcal{L}) \backslash\{m\}}\right|=1$. It is not hard to see that $\Gamma_{S}(\mathcal{L} \backslash \mathcal{J}(\mathcal{L}))$ is a complete 3-partite graph such that at least two parts of it has exactly one element. Therefore $\Gamma_{S}(\mathcal{L} \backslash \mathcal{J}(\mathcal{L}))$ is planar. Now, by Proposition $4.11, \Gamma_{S}(\mathcal{L})$ is planar.

Now, suppose that $|\operatorname{Coatom}(\mathcal{L})|=3$ and put $\operatorname{Coatom}(\mathcal{L})=\left\{m, m^{\prime}, m^{\prime \prime}\right\}$. Also, assume that the conditions (a) and (b) hold. It is easy to see that the subgraph $\Gamma_{S}(\mathcal{L} \backslash \mathcal{J}(\mathcal{L}))$ on $\{1\} \cup L_{\left\{m^{\prime}, m^{\prime \prime}\right\}}^{m} \cup L_{\left\{m, m^{\prime \prime}\right\}}^{m^{\prime}} \cup L_{\left\{m, m^{\prime}\right\}}^{m^{\prime \prime}}$ is a complete 4-partite graph such that at least three parts of it has exactly one element. Now, by condition (b), it is easy to see that $\Gamma_{S}(\mathcal{L} \backslash \mathcal{J}(\mathcal{L}))$ is planar. Hence, by Proposition $4.11, \Gamma_{S}(\mathcal{L})$ is planar.

Acknowledgements The authors are deeply grateful to the referees for careful reading of the manuscript and helpful suggestions.

Open Access This article is distributed under the terms of the Creative Commons Attribution License which permits any use, distribution, and reproduction in any medium, provided the original author(s) and the source are credited. 


\section{References}

1. Akbari, S., Kiani, D., Mohammadi, F., Moradi, S.: The total graph and regular graph of a commutative ring. J. Pure Appl. Algebra 213, 2224-2228 (2009)

2. Anderson, D.F., Badawi, A.: The total graph of a commutative ring. J. Algebra 320, 2706-2719 (2008)

3. Anderson, D.F., Livingston, P.S.: The zero-divisor graph of a commutative ring. J. Algebra 217, 434-447 (1999)

4. Ashrafi, N., Maimani, H.R., Pournaki, M.R., Yassemi, S.: Unit graphs associated with rings. Comm. Algebra 38, 2851-2871 (2010)

5. Barati, Z., Khashyarmanesh, K., Mohammadi, F., Nafar, Kh.: On the associated graphs to a commutative ring. J. Algebra Appl. 11:1250037 (17 pages) (2012)

6. Beck, I.: Coloring of commutative rings. J. Algebra 116, 208-226 (1998)

7. Bondy, J.A., Murty, U.S.R.: Graph Theory with applications. American Elsevier, New York (1976)

8. Donnellan, T.: Lattice Theory. Pergamon Press, Oxford (1968) 